\title{
A type of uncertain differential equations with analytic solution
}

Kai Yao

Correspondence: yaokai@ucas.ac.cn School of Management, University of Chinese Academy of Sciences, Beijing 100190, China

\begin{abstract}
Uncertain differential equation is a type of differential equation driven by the Liu process. So far, an analytic solution of linear uncertain differential equation has been obtained. This paper aims at proposing a method to solve a type of nonlinear uncertain differential equation.
\end{abstract}

Keywords: Uncertainty theory; Uncertain differential equation; Liu process

\section{Introduction}

Uncertainty theory, as a branch of axiomatic mathematics dealing with human's belief degree, was founded by Liu [1] in 2007 and refined by Liu [2] in 2010. During the past 6 years, many researchers have contributed in this area. For example, Peng and Iwamura [3] gave a sufficient condition for the uncertainty distribution of an uncertain variable. Liu and $\mathrm{Ha}$ [4] gave a formula to calculate the expected value of a function of multiple uncertain variables. Chen and Dai [5] showed that a normal uncertain variable has the maximum entropy given the expected value and variance. Especially, Liu [6] proposed uncertain programming as a type of mathematical programming involving uncertain variables.

In order to model the evolution of an uncertain phenomenon, Liu [7] proposed a concept of uncertain process. Meanwhile, Liu [7] gave an uncertain renewal process as an example. After that, Liu [2] proposed an uncertain renewal reward process, and Yao and $\mathrm{Li}$ [8] proposed an uncertain alternating renewal process. In addition, Zhang et al. [9] proposed an uncertain delayed renewal process. In 2009, Liu [10] mathematically defined a type of uncertain process, named canonical Liu process, which has independent and stationary uncertain normal increments and of which almost all the sample paths are Lipschitz continuous. In addition, Liu [11] proved the extreme value theorems for an independent increment uncertain process.

In 2009, Liu [10] founded an uncertain calculus to deal with the integral and differential of an uncertain process with respect to Liu process, which are called Liu integral and Liu differential afterwards. Then Liu and Yao [12] studied an uncertain integral with respect to multiple Liu processes. After that, Chen and Ralescu [13] proposed an uncertain integral with respect to the general Liu process. Inspired by the Liu integral, Yao [14] proposed an uncertain calculus with respect to an uncertain renewal process.

(c) 2013 Yao; licensee Springer. This is an Open Access article distributed under the terms of the Creative Commons Attribution License (http://creativecommons.org/licenses/by/2.0), which permits unrestricted use, distribution, and reproduction in any medium, provided the original work is properly cited. 
Uncertain differential equation was first proposed by Liu [7] as a type of differential equation driven by Liu process. Then Chen and Liu [15] gave a sufficient condition for an uncertain differential equation having a unique solution. After that, Gao [16] provided an existence and unique theorem under weaker conditions. In 2013, Yao et al. [17] gave a sufficient condition for it being stable. After that, Sheng [18] studied the stability in $p$ th moment.

Nowadays, uncertain differential equation has been applied to many areas especially in finance. In 2009, Liu [10] assumed that the stock price follows a geometric Liu process in the short run and proposed a stock model in an uncertain environment. After that, Chen [19] derived its American option formulae. In 2011, Peng and Yao [20] proposed another model to describe the stock price displaying a mean-reverting property in the long run via uncertain differential equation. In 2013, Chen et al. [21] proposed a stock model with periodic dividends. Assuming that the interest rate follows an uncertain differential equation, Chen and Gao [22] presented an uncertain interest rate model and calculated its zerocoupon bond. Besides, Liu et al. [23] proposed an uncertain currency model via uncertain differential equation. For more recent developments in uncertain finance, please refer to Liu [24].

In 2010, Chen and Liu [15] gave an analytic solution for the linear uncertain differential equation. Then Liu [25] proposed a method to solve a special type of nonlinear uncertain differential equation. In addition, some numerical methods were designed to calculate the uncertainty distributions of the solution and the extreme values of the solution by Yao and Chen [26] and Yao [27]. In this paper, we will give an analytic method to solve another special type of uncertain differential equation. The rest of this paper is organized as follows. Some concepts and theorems about uncertainty theory, uncertain calculus, and uncertain differential equation will be introduced in the 'Uncertainty theory' section, 'Uncertain calculus' section, and 'Uncertain differential equation' section, respectively. Then a special type of uncertain differential equation is solved in the 'An analytic method' section. Finally, some remarks are made in the 'Conclusions' section.

\section{Uncertainty theory}

This section will briefly introduce some basic concepts in uncertainty theory, including uncertainty space, uncertain variable, independence, and the operational law.

Definition 1. (Liu [1]) Let $\mathcal{L}$ be a $\sigma$-algebra on a nonempty set $\Gamma$. A set function $\mathcal{M}$ : $\mathcal{L} \rightarrow[0,1]$ is called an uncertain measure if it satisfies the following axioms:

Axiom 1. (Normality Axiom) $\mathcal{M}\{\Gamma\}=1$ for the universal set $\Gamma$.

Axiom 2. (Duality Axiom) $\mathcal{M}\{\Lambda\}+\mathcal{M}\left\{\Lambda^{c}\right\}=1$ for any event $\Lambda$.

Axiom 3. (Subadditivity Axiom) For every countable sequence of events $\Lambda_{1}, \Lambda_{2}, \ldots$, we have

$$
\mathcal{M}\left\{\bigcup_{i=1}^{\infty} \Lambda_{i}\right\} \leq \sum_{i=1}^{\infty} \mathcal{M}\left\{\Lambda_{i}\right\}
$$

Besides, the product uncertain measure on the product $\sigma$-algebre $\mathcal{L}$ is defined by Liu [10] as follows: 
Axiom 4. (Product Axiom) Let $\left(\Gamma_{k}, \mathcal{L}_{k}, \mathcal{M}_{k}\right)$ be uncertainty spaces for $k=1,2, \ldots$ Then the product uncertain measure $\mathcal{M}$ on the product $\sigma$-algebra satisfies

$$
\mathcal{M}\left\{\prod_{i=1}^{\infty} \Lambda_{k}\right\}=\bigwedge_{k=1}^{\infty} \mathcal{M}_{k}\left\{\Lambda_{k}\right\}
$$

where $\Lambda_{k}$ are arbitrarily chosen events from $\mathcal{L}_{k}$ for $k=1,2, \ldots$, respectively.

In order to represent the quantities with uncertainty, an uncertain variable was proposed as a real valued function on an uncertainty space.

Definition 2. (Liu [1]) An uncertain variable $\xi$ is a measurable function from an uncertainty space $(\Gamma, \mathcal{L}, \mathcal{M})$ to the set of real numbers, i.e., for any Borel set $B$, the set $\{\gamma \mid \xi(\gamma) \in B\}$ is an event in $\mathcal{L}$.

The uncertainty distribution $\Phi$ of an uncertain variable is defined by $\Phi(x)=\mathcal{M}\{\xi \leq x\}$ for any real number $x$. The expected value of an uncertain variable $\xi$ is

$$
E[\xi]=\int_{0}^{+\infty} \mathcal{M}\{\xi \geq r\} \mathrm{d} r-\int_{-\infty}^{0} \mathcal{M}\{\xi \leq r\} \mathrm{d} r
$$

provided that at least one of the two integrals is finite, and the variance of $\xi$ is

$$
V[\xi]=E\left[(\xi-E[\xi])^{2}\right] .
$$

An uncertain variable $\xi$ is said to be normal if it has an uncertainty distribution

$$
\Phi(x)=\left(1+\exp \left(\frac{\pi(e-x)}{\sqrt{3} \sigma}\right)\right)^{-1}, \quad x \in \mathfrak{R}
$$

where $e$ and $\sigma^{2}$ are the expected value and variance of $\xi$, respectively.

Definition 3. (Liu [10]) The uncertain variables $\xi_{1}, \xi_{2}, \ldots, \xi_{n}$ are said to be independent if

$$
\mathcal{M}\left\{\bigcap_{i=1}^{n}\left(\xi_{i} \in B_{i}\right)\right\}=\bigwedge_{i=1}^{n} \mathcal{M}\left\{\xi_{i} \in B_{i}\right\}
$$

for any Borel sets $B_{1}, B_{2}, \ldots, B_{n}$.

Since the definition of independence for uncertain variables is quite different from that for random variables, the operational law of uncertain variables is also different.

Theorem 1. (Liu [2]) Let $\xi_{1}, \xi_{2}, \ldots, \xi_{n}$ be independent uncertain variables with uncertainty distributions $\Phi_{1}, \Phi_{2}, \ldots, \Phi_{n}$, respectively. If $f\left(x_{1}, x_{2}, \ldots, x_{n}\right)$ is strictly increasing with respect to $x_{1}, x_{2}, \ldots, x_{m}$ and strictly decreasing with respect to $x_{m+1}, x_{m+2}, \ldots, x_{n}$, then $\xi=f\left(\xi_{1}, \xi_{2}, \ldots, \xi_{n}\right)$ is an uncertain variable with an inverse uncertainty distribution

$$
\Phi^{-1}(r)=f\left(\Phi_{1}^{-1}(r), \ldots, \Phi_{m}^{-1}(r), \Phi_{m+1}^{-1}(1-r), \ldots, \Phi_{n}^{-1}(1-r)\right) .
$$

\section{Uncertain calculus}

An uncertain process is essentially a sequence of uncertain variables indexed by time or space. As one of the most important types of uncertain processes, the canonical Liu process is defined as follows: 
Definition 4. (Liu [10]) An uncertain process $C_{t}$ is called a canonical Liu process if:

1. $C_{0}=0$ and almost all sample paths are Lipschitz continuous.

2. $C_{t}$ has stationary and independent increments.

3. Every increment $C_{s+t}-C_{s}$ is a normal uncertain variable $\mathcal{N}(0, t)$ with expected value 0 and variance $t^{2}$ whose uncertainty distribution is

$$
\Phi_{t}(x)=\left(1+\exp \left(-\frac{\pi x}{\sqrt{3} t}\right)\right)^{-1}, \quad x \in \Re .
$$

Based on the canonical Liu process, Liu [10] proposed an uncertain integral of an uncertain process with respect to the canonical Liu process and thus founded a theory of uncertain calculus.

Definition 5. (Liu [10]) Let $X_{t}$ be an uncertain process and $C_{t}$ be a canonical Liu process. For any partition of closed interval $[a, b]$ with $a=t_{1}<t_{2}<\cdots<t_{k+1}=b$, the mesh is written as

$$
\Delta=\max _{1 \leq i \leq k}\left|t_{i+1}-t_{i}\right| .
$$

Then the Liu integral of $X_{t}$ is defined by

$$
\int_{a}^{b} X_{t} \mathrm{~d} C_{t}=\lim _{\Delta \rightarrow 0} \sum_{i=1}^{k} X_{t_{i}} \cdot\left(C_{t_{i+1}}-C_{t_{i}}\right)
$$

provided that the limit exists almost surely and is finite.

For example, the Liu integral of an integrable real function $f(t)$ is

$$
\int_{0}^{t} f(s) \mathrm{d} C_{s} \sim\left(0, \int_{0}^{t}|f(s)| \mathrm{d} s\right)
$$

at each time $t$.

Definition 6. (Liu [10]) Let $X_{t}$ be an uncertain process and $N_{t}$ be an uncertain renewal process. Then the Yao integral of $X_{t}$ is defined by

$$
\int_{a}^{b} X_{t} \mathrm{~d} N_{t}=\sum_{a<t \leq b} X_{t-}\left(N_{t}-N_{t-}\right)
$$

provided that the sum exists almost surely and is finite.

For a continuously differentiable function $h(t, c, n)$, the uncertain process $Z_{t}=h\left(t, C_{t}, N_{t}\right)$ has an uncertain differential

$$
\mathrm{d} Z_{t}=\frac{\partial h}{\partial t}\left(t, C_{t}, N_{t}\right) \mathrm{d} t+\frac{\partial h}{\partial c}\left(t, C_{t}, N_{t}\right) \mathrm{d} C_{t}+h\left(t, C_{t}, N_{t}\right)-h\left(t, C_{t}, N_{t-}\right) .
$$

For example, the uncertain differential of an uncertain process $X_{t}=\mu t+\sigma C_{t}+\gamma N_{t}$ is

$$
\mathrm{d} X_{t}=\mu \mathrm{d} Z_{t}+\sigma \mathrm{d} C_{t}+\gamma\left(N_{t}-N_{t-}\right),
$$

and the uncertain differential of an uncertain process $Y_{t}=t C_{t} N_{t}$ is

$$
\mathrm{d} Y_{t}=C_{t} N_{t} \mathrm{~d} t+t N_{t} \mathrm{~d} C_{t}+t C_{t}\left(N_{t}-N_{t-}\right) .
$$




\section{Uncertain differential equation}

Definition 7. (Liu [7]) Suppose $C_{t}$ is a canonical Liu process, and $f$ and $g$ are two given functions. Then

$$
\mathrm{d} X_{t}=f\left(t, X_{t}\right) \mathrm{d} t+g\left(t, X_{t}\right) \mathrm{d} C_{t}
$$

is called an uncertain differential equation.

In 2010, Chen and Liu [15] solved the linear uncertain differential equation

$$
\mathrm{d} X_{t}=\left(u_{1 t} X_{t}+u_{2 t}\right) \mathrm{d} t+\left(v_{1 t} X_{t}+v_{2 t}\right) \mathrm{d} C_{t}
$$

and obtained a solution

$$
X_{t}=Y_{t}\left(X_{0}+\int_{0}^{t} \frac{u_{2 s}}{Y_{s}} \mathrm{~d} s+\int_{0}^{t} \frac{v_{2 s}}{Y_{s}} \mathrm{~d} C_{s}\right)
$$

where

$$
Y_{t}=\exp \left(\int_{0}^{t} u_{1 s} \mathrm{~d} s+\int_{0}^{t} v_{1 s} \mathrm{~d} C_{s}\right) .
$$

After that, Liu [25] provided a method to solve the following types of uncertain differential equations:

$$
\begin{aligned}
\mathrm{d} X_{t} & =f\left(t, X_{t}\right) \mathrm{d} t+\sigma_{t} X_{t} \mathrm{~d} C_{t}, \\
\mathrm{~d} X_{t} & =\mu_{t} X_{t} \mathrm{~d} t+g\left(t, X_{t}\right) \mathrm{d} C_{t},
\end{aligned}
$$

and showed that uncertain differential equation (2) has a solution $X_{t}=Y_{t}^{-1} Z_{t}$ where

$$
Y_{t}=\exp \left(-\int_{0}^{t} \sigma_{s} \mathrm{~d} C_{s}\right)
$$

and $Z_{t}$ solves uncertain differential equation $\mathrm{d} Z_{t}=Y_{t} f\left(t, Y_{t}^{-1} Z_{t}\right) \mathrm{d} t$ with an initial value $Z_{0}=X_{0}$, and uncertain differential equation (3) has a solution $X_{t}=Y_{t}^{-1} Z_{t}$ where

$$
Y_{t}=\exp \left(-\int_{0}^{t} \alpha_{s} \mathrm{~d} s\right)
$$

and $Z_{t}$ solves uncertain differential equation $\mathrm{d} Z_{t}=Y_{t} g\left(t, Y_{t}^{-1} Z_{t}\right) \mathrm{d} C_{t}$ with an initial value $Z_{0}=X_{0}$.

\section{An analytic method}

In this section, we will propose an analytic method to solve uncertain differential equations

$$
\mathrm{d} X_{t}=f\left(t, X_{t}\right) \mathrm{d} t+\sigma_{t} \mathrm{~d} C_{t}
$$

and

$$
\mathrm{d} X_{t}=\mu_{t} \mathrm{~d} t+g\left(t, X_{t}\right) \mathrm{d} C_{t} .
$$

\section{Model I}

Theorem 2. Let fbe a function of two variables, and $\sigma_{t}$ be an integrable function on $\mathfrak{R}^{+}$. Then the uncertain differential equation

$$
\mathrm{d} X_{t}=f\left(t, X_{t}\right) \mathrm{d} t+\sigma_{t} \mathrm{~d} C_{t}
$$


has a solution

$$
X_{t}=Y_{t}+Z_{t}
$$

where

$$
Y_{t}=\int_{0}^{t} \sigma_{s} \mathrm{~d} C_{s}
$$

and $Z_{t}$ is the solution of uncertain differential equation

$$
\mathrm{d} Z_{t}=f\left(t, Y_{t}+Z_{t}\right) \mathrm{d} t, \quad Z_{0}=X_{0} .
$$

Proof. The uncertain process $Y_{t}$ has an uncertain differential

$$
\mathrm{d} Y_{t}=\mu_{t} \mathrm{~d} t .
$$

Then we have

$$
\mathrm{d}\left(X_{t}-Y_{t}\right)=\mathrm{d} X_{t}-\mathrm{d} Y_{t}=f\left(t, X_{t}\right) \mathrm{d} t+\sigma_{t} \mathrm{~d} C_{t}-\sigma_{t} \mathrm{~d} C_{t} .
$$

That is,

$$
\mathrm{d}\left(X_{t}-Y_{t}\right)=f\left(t, X_{t}\right) \mathrm{d} t .
$$

Defining $Z_{t}=X_{t}-Y_{t}$, we obtain $X_{t}=Y_{t}+Z_{t}$ and $\mathrm{d} Z_{t}=f\left(t, Y_{t}+Z_{t}\right) \mathrm{d} t$. Furthermore, since $Y_{0}=0$, the initial value $Z_{0}$ is just $X_{0}$. The theorem is verified.

Remark 1. If $\sigma_{t}$ degenerates to a constant $\sigma$, then $Y_{t}=\sigma C_{t}$. The uncertain differential equation

$$
\mathrm{d} X_{t}=f\left(t, X_{t}\right) \mathrm{d} t+\sigma \mathrm{d} C_{t}
$$

has a solution

$$
X_{t}=Z_{t}+\sigma C_{t}
$$

where $Z_{t}$ solves the uncertain differential equation

$$
\mathrm{d} Z_{t}=f\left(t, Z_{t}+\sigma C_{t}\right) \mathrm{d} t, \quad Z_{0}=X_{0} .
$$

Example 1. Let $m_{t}, \mu_{t}$, and $\sigma_{t}$ be some real functions on $\Re$. Consider the uncertain differential equation

$$
\mathrm{d} X_{t}=\left(m_{t}-\mu_{t} X_{t}\right) \mathrm{d} t+\sigma_{t} \mathrm{~d} C_{t} .
$$

At first, we have

$$
Y_{t}=\int_{0}^{t} \sigma_{s} \mathrm{~d} C_{s}
$$

and $Z_{t}$ solves the uncertain differential equation

$$
\mathrm{d} Z_{t}=\left(m_{t}-\mu_{t}\left(\int_{0}^{t} \sigma_{s} \mathrm{~d} s+Z_{t}\right)\right) \mathrm{d} t
$$

so

$$
\mathrm{d} Z_{t}+\mu_{t} Z_{t} \mathrm{~d} t=\left(m_{t}-\mu_{t} \int_{0}^{t} \sigma_{s} \mathrm{~d} C_{s}\right) \mathrm{d} t .
$$

It follows from the fundamental theorem of uncertain calculus that

$$
\mathrm{d}\left(\exp \left(\int_{0}^{t} \mu_{s} \mathrm{~d} s\right) Z_{t}\right)=\exp \left(\int_{0}^{t} \mu_{s} \mathrm{~d} s\right)\left(m_{t}-\mu_{t} \int_{0}^{t} \sigma_{s} \mathrm{~d} C_{s}\right) \mathrm{d} t .
$$


That is,

$$
\exp \left(\int_{0}^{t} \mu_{s} \mathrm{~d} s\right) Z_{t}-Z_{0}=\int_{0}^{t} \exp \left(\int_{0}^{s} \mu_{\nu} \mathrm{d} v\right)\left(m_{s}-\mu_{s} \int_{0}^{s} \sigma_{\nu} \mathrm{d} C_{v}\right) \mathrm{d} s .
$$

As a result,

$$
\begin{aligned}
Z_{t} & =\exp \left(-\int_{0}^{t} \mu_{s} \mathrm{~d} s\right) Z_{0}+\int_{0}^{t} \exp \left(-\int_{s}^{t} \mu_{\nu} \mathrm{d} v\right)\left(m_{s}-\mu_{s} \int_{0}^{s} \sigma_{\nu} \mathrm{d} C_{\nu}\right) \mathrm{d} s \\
& =\exp \left(-\int_{0}^{t} \mu_{s} \mathrm{~d} s\right) X_{0}+\int_{0}^{t} \exp \left(-\int_{s}^{t} \mu_{\nu} \mathrm{d} v\right)\left(m_{s}-\mu_{s} \int_{0}^{s} \sigma_{\nu} \mathrm{d} C_{\nu}\right) \mathrm{d} s .
\end{aligned}
$$

By Theorem 2, we have

$X_{t}=Y_{t}+Z_{t}=\exp \left(-\int_{0}^{t} \mu_{s} \mathrm{~d} s\right) X_{0}+\int_{0}^{t} \sigma_{s} \mathrm{~d} C_{s}+\int_{0}^{t} \exp \left(-\int_{s}^{t} \mu_{\nu} \mathrm{d} \nu\right)\left(m_{s}-\mu_{s} \int_{0}^{s} \sigma_{\nu} \mathrm{d} C_{v}\right) \mathrm{d} s$

Example 2. Let $\mu$ and $\sigma$ be real numbers with $\mu \neq 0$. Consider the uncertain differential equation

$$
\mathrm{d} X_{t}=\mu \exp \left(X_{t}\right) \mathrm{d} t+\sigma \mathrm{d} C_{t} .
$$

At first, we have $Y_{t}=\sigma C_{t}$ and $Z_{t}$ satisfies the uncertain differential equation

$$
\mathrm{d} Z_{t}=\mu \exp \left(\sigma C_{t}+Z_{t}\right) \mathrm{d} t
$$

so

$$
\exp \left(-Z_{t}\right) \mathrm{d} Z_{t}=\mu \exp \left(\sigma C_{t}\right) \mathrm{d} t
$$

It follows from the fundamental theorem of uncertain calculus that

$$
\mathrm{d} \exp \left(-Z_{t}\right)=-\mu \exp \left(\sigma C_{t}\right) \mathrm{d} t
$$

That is,

$$
\exp \left(-Z_{t}\right)-\exp \left(-Z_{0}\right)=-\mu \int_{0}^{t} \exp \left(\sigma C_{s}\right) \mathrm{d} s
$$

As a result,

$$
Z_{t}=Z_{0}-\ln \left(1-\mu \int_{0}^{t} \exp \left(Z_{0}+\sigma C_{s}\right) \mathrm{d} s\right)=X_{0}-\ln \left(1-\mu \int_{0}^{t} \exp \left(X_{0}+\sigma C_{s}\right) \mathrm{d} s\right) .
$$

By Theorem 2, we have

$$
X_{t}=Y_{t}+Z_{t}=X_{0}+\sigma C_{t}-\ln \left(1-\mu \int_{0}^{t} \exp \left(X_{0}+\sigma C_{s}\right) \mathrm{d} s\right) \text {. }
$$

\section{Model II}

Theorem 3. Let $\mu_{t}$ be an integrable function on $\mathfrak{R}^{+}$, and $g$ be a function of two variables.

Then the uncertain differential equation

$$
\mathrm{d} X_{t}=\mu_{t} \mathrm{~d} t+g\left(t, X_{t}\right) \mathrm{d} C_{t}
$$

has a solution

$$
X_{t}=Y_{t}+Z_{t}
$$

where

$$
Y_{t}=\int_{0}^{t} \mu_{s} \mathrm{~d} s
$$


and $Z_{t}$ is the solution of uncertain differential equation

$$
\mathrm{d} Z_{t}=g\left(t, Y_{t}+Z_{t}\right) \mathrm{d} C_{t}, \quad Z_{0}=X_{0} .
$$

Proof. The uncertain process $Y_{t}$ has an uncertain differential

$$
\mathrm{d} Y_{t}=\mu_{t} \mathrm{~d} t .
$$

Then we have

$$
\mathrm{d}\left(X_{t}-Y_{t}\right)=\mathrm{d} X_{t}-\mathrm{d} Y_{t}=\mu_{t} \mathrm{~d} t+g\left(t, X_{t}\right) \mathrm{d} C_{t}-\mu_{t} \mathrm{~d} C_{t} .
$$

That is,

$$
\mathrm{d}\left(X_{t}-Y_{t}\right)=g\left(t, X_{t}\right) \mathrm{d} C_{t}
$$

Defining $Z_{t}=X_{t}-Y_{t}$, we obtain $X_{t}=Y_{t}+Z_{t}$ and $\mathrm{d} Z_{t}=g\left(t, Y_{t}+Z_{t}\right) \mathrm{d} C_{t}$. Furthermore, since $Y_{0}=0$, the initial value $Z_{0}$ is just $X_{0}$. The theorem is verified.

Remark 2. If $\mu_{t}$ degenerates to a constant $\mu$, then $Y_{t}=\mu t$, and the uncertain differential equation

$$
\mathrm{d} X_{t}=\mu \mathrm{d} t+g\left(t, X_{t}\right) \mathrm{d} C_{t}
$$

has a solution

$$
X_{t}=Z_{t}+\mu t
$$

where $Z_{t}$ solves the uncertain differential equation

$$
\mathrm{d} Z_{t}=g\left(t, Z_{t}+\mu t\right) \mathrm{d} t, \quad Z_{0}=X_{0} .
$$

Example 3. Let $m_{t}, \mu_{t}$, and $\sigma_{t}$ be some real functions on $\Re$. Consider the uncertain differential equation

$$
\mathrm{d} X_{t}=\mu_{t} \mathrm{~d} t+\left(m_{t}-\sigma_{t} X_{t}\right) \mathrm{d} C_{t} .
$$

At first, we have

$$
Y_{t}=\int_{0}^{t} \mu_{s} \mathrm{~d} s
$$

and $Z_{t}$ solves the uncertain differential equation

$$
\mathrm{d} Z_{t}=\left(m_{t}-\sigma_{t}\left(\int_{0}^{t} \mu_{s} \mathrm{~d} s+Z_{t}\right)\right) \mathrm{d} C_{t},
$$

so

$$
\mathrm{d} Z_{t}+\sigma_{t} Z_{t} \mathrm{~d} C_{t}=\left(m_{t}-\sigma_{t} \int_{0}^{t} \mu_{s} \mathrm{~d} s\right) \mathrm{d} C_{t} .
$$

It follows from the fundamental theorem of uncertain calculus that

$$
\mathrm{d}\left(\exp \left(\int_{0}^{t} \sigma_{s} \mathrm{~d} C_{s}\right) Z_{t}\right)=\exp \left(\int_{0}^{t} \sigma_{s} \mathrm{~d} C_{s}\right)\left(m_{t}-\sigma_{t} \int_{0}^{t} \mu_{s} \mathrm{~d} s\right) \mathrm{d} C_{t} .
$$

That is,

$$
\exp \left(\int_{0}^{t} \sigma_{s} \mathrm{~d} C_{s}\right) Z_{t}-Z_{0}=\int_{0}^{t} \exp \left(\int_{0}^{s} \sigma_{\nu} \mathrm{d} C_{\nu}\right)\left(m_{s}-\sigma_{s} \int_{0}^{s} \mu_{\nu} \mathrm{d} v\right) \mathrm{d} C_{s} .
$$


As a result,

$$
\begin{aligned}
Z_{t} & =\exp \left(-\int_{0}^{t} \sigma_{s} \mathrm{~d} C_{s}\right) Z_{0}+\int_{0}^{t} \exp \left(-\int_{s}^{t} \sigma_{\nu} \mathrm{d} C_{\nu}\right)\left(m_{s}-\sigma_{s} \int_{0}^{s} \mu_{\nu} \mathrm{d} v\right) \mathrm{d} C_{s} \\
& =\exp \left(-\int_{0}^{t} \sigma_{s} \mathrm{~d} C_{s}\right) X_{0}+\int_{0}^{t} \exp \left(-\int_{s}^{t} \sigma_{\nu} \mathrm{d} C_{\nu}\right)\left(m_{s}-\sigma_{s} \int_{0}^{s} \mu_{\nu} \mathrm{d} v\right) \mathrm{d} C_{s} .
\end{aligned}
$$

By Theorem 3, we have

$$
X_{t}=Y_{t}+Z_{t}=\exp \left(-\int_{0}^{t} \sigma_{s} \mathrm{~d} C_{s}\right) X_{0}+\int_{0}^{t} \mu_{s} \mathrm{~d} s+\int_{0}^{t} \exp \left(-\int_{s}^{t} \sigma_{\nu} \mathrm{d} C_{\nu}\right)\left(m_{s}-\sigma_{s} \int_{0}^{s} \mu_{\nu} \mathrm{d} v\right) \mathrm{d} C_{s} .
$$

Example 4. Let $\mu$ and $\sigma$ be real numbers with $\sigma \neq 0$. Consider the uncertain differential equation

$$
\mathrm{d} X_{t}=\mu \mathrm{d} t+\sigma \exp \left(X_{t}\right) \mathrm{d} C_{t} .
$$

At first, we have $Y_{t}=\mu t$ and $Z_{t}$ satisfies the uncertain differential equation

$$
\mathrm{d} Z_{t}=\sigma \exp \left(\mu t+Z_{t}\right) \mathrm{d} C_{t},
$$

so

$$
\exp \left(-Z_{t}\right) \mathrm{d} Z_{t}=\sigma \exp (\mu t) \mathrm{d} C_{t} .
$$

It follows from the fundamental theorem of uncertain calculus that

$$
\mathrm{d} \exp \left(-Z_{t}\right)=-\sigma \exp (\mu t) \mathrm{d} C_{t} .
$$

That is,

$$
\exp \left(-Z_{t}\right)-\exp \left(-X_{0}\right)=-\sigma \int_{0}^{t} \exp (\mu s) \mathrm{d} C_{s}
$$

As a result,

$$
Z_{t}=Z_{0}-\ln \left(1-\sigma \int_{0}^{t} \exp \left(Z_{0}+\mu s\right) \mathrm{d} C_{s}\right)=X_{0}-\ln \left(1-\sigma \int_{0}^{t} \exp \left(X_{0}+\mu s\right) \mathrm{d} C_{s}\right)
$$

By Theorem 3, we have

$$
X_{t}=Y_{t}+Z_{t}=X_{0}+\mu t-\ln \left(1-\sigma \int_{0}^{t} \exp \left(X_{0}+\mu s\right) \mathrm{d} C_{s}\right)
$$

\section{Conclusions}

This paper proposed a method to solve a special type of uncertain differential equation and employed some examples to illustrate the method.

\section{Acknowledgements}

This work was supported by National Natural Science Foundation of China Grant Nos. 61273044 and 91224008.

Received: 25 August 2013 Accepted: 10 October 2013

Published: 30 October 2013

\section{References}

1. Liu, B: Uncertainty Theory. 2nd ed. Springer, Berlin (2007)

2. Liu, B: Uncertainty Theory: A Branch of Mathematics for Modeling Human Uncertainty. Springer, Berlin (2010)

3. Peng, ZX, Iwamura, K: A sufficient and necessary condition of uncertainty distribution. J. Interdiscip. Math. 13(3), 277-285 (2010)

4. Liu, YH, Ha, M: Expected value of function of uncertain variables. J. Uncertain Syst. 4(3), 181-186 (2010)

5. Chen, X, Dai, W: Maximum entropy principle for uncertain variables. Int. J. Fuzzy Syst. 13(3), 232-236 (2011)

6. Liu, B: Theory and Practice of Uncertain Programming. 2nd ed. Springer, Berlin (2009)

7. Liu, B: Fuzzy process, hybrid process and uncertain process. J. Uncertain Syst. 2(1), 3-16 (2008)

8. Yao, K, Li, X: Uncertain alternating renewal process and its application. IEEE Trans. Fuzzy Syst. 20(6), 1154-1160 (2012)

9. Zhang, $X$, Ning, $Y$, Meng, G: Delayed renewal process with uncertain interarrival times. Fuzzy Optimization Decis Making 12(1), 79-87 (2013) 
10. Liu, B: Some research problems in uncertainty theory. J. Uncertain Syst. 3(1), 3-10 (2009)

11. Liu, B: Extreme value theorems of uncertain process with application to insurance risk model. Soft Comput. 17(4), 549-556 (2013)

12. Liu, B, Yao, K: Uncertain integral with respect to multiple canonical processes. J. Uncertain Syst. 6(4), 250-255 (2012)

13. Chen, X, Ralescu, DA: Liu process and uncertain calculus. J. Uncertainty Anal. Appl. 1, Article 3 (2013)

14. Yao, K: Uncertain calculus with renewal process. Fuzzy Optimization Decis. Making 11(3), 285-297 (2012)

15. Chen, $X, L i u, B$ : Existence and uniqueness theorem for uncertain differential equations. Fuzzy Optimization Decis Making 9(1), 69-81 (2010)

16. Gao, Y: Existence and uniqueness theorem on uncertain differential equations with local Lipschitz condition. J. Uncertain Syst. 6(3), 223-232 (2012)

17. Yao, K, Gao, J, Gao, Y: Some stability theorems of uncertain differential equation. Fuzzy Optimization Decis Making 12(1), 3-13 (2013)

18. Sheng, Y: Stability in the $\mathrm{p}$-th moment for uncertain differential equation. J. Intell. Fuzzy Syst. (2013, in press)

19. Chen, $X$ : American option pricing formula for uncertain financial market. Int. J. Oper. Res. 8(2), 32-37 (2011)

20. Peng, J, Yao, K: A new option pricing model for stocks in uncertainty markets. Int. J. Oper. Res. 8(2), 18-26 (2011)

21. Chen, $X$, Liu, YH, Ralescu, DA: Uncertain stock model with periodic dividends. Fuzzy Optimization Decis Making 12(1), 111-123 (2013)

22. Chen, $X$, Gao, Y: Uncertain term structure model of interest rate. Soft Comput. 17(4), 597-604 (2013)

23. Liu, YH, Chen, X, Ralescu, DA: Uncertain currency model and currency option pricing. Int. J. Intell. Syst. (2013, in press)

24. Liu, B: Toward uncertain finance theory. J. Uncertainty Anal. Appl. 1, Article 1 (2013)

25. Liu, YH: An analytic method for solving uncertain differential equations. J. Uncertain Syst. 6(4), 244-249 (2012)

26. Yao, K, Chen, X: A numerical method for solving uncertain differential equations. J. Intell. Fuzzy Syst. 25(3), 825-832 (2013)

27. Yao, K: Extreme values and integral of solution of uncertain differential equation. J. Uncertainty Anal. Appl. 1, Article 2 (2013)

doi:10.1186/2195-5468-1-8

Cite this article as: Yao: A type of uncertain differential equations with analytic solution. Journal of Uncertainty Analysis and Applications 2013 1:8.

\section{Submit your manuscript to a SpringerOpen ${ }^{\circ}$ journal and benefit from:}

- Convenient online submission

- Rigorous peer review

- Immediate publication on acceptance

- Open access: articles freely available online

- High visibility within the field

- Retaining the copyright to your article

Submit your next manuscript at $\boldsymbol{\triangleright}$ springeropen.com 